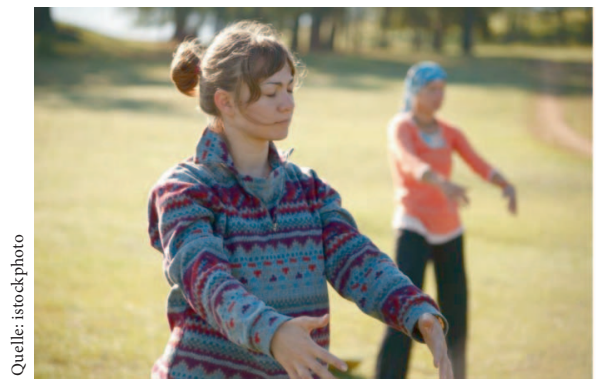

Für viele Menschen mit einem hohen Risiko für Herz-Kreislauf-Erkrankungen ist es schwer, ihren bewegungsarmen Alltag umzustellen, um mehr Aktivitäten in den Tagesablauf zu integrieren. Deshalb suchen viele Ärzte und Gesundheitstrainer nach neuen Methoden, um mit attraktiven Sportarten die Compliance der Patientinnen und Patienten zu verbessern. Dabei geht es vor allem darum, ihre Motivation aufrechtzuerhalten und sie nicht zu überfordern. Eine Möglichkeit, Patienten mit stark reduzierter körperlicher Leistungsfähigkeit zu mehr Bewegung zu verhelfen und die anderen länger bei der Stange $\mathrm{zu}$ halten, könnten die chinesischen Bewegungstrainings Qigong oder TaiChi sein. Wie kürzlich in einigen Studien belegt wurde, haben die traditionellen chinesischen Bewegungstrainings eine Reihe von Vorzügen [1]. Tai-Chi ist eine sehr alte Konditionsund Körperbeherrschungstechnik, die sich in China auch heute noch grosser Beliebtheit erfreut. Dabei wird der Körper nicht auf Hochleistung gebracht, sondern der Stoffwechsel und Puls nur leicht dosiert gesteigert. Körperliches Training, das den Pulsschlag auf $50-70 \%$ der normalen Reserven ansteigen lässt und regelmässig betrieben wird, zeigt Erfolg bei der Reduktion von Risikofaktoren für Herz-Kreislauf-Erkrankungen. Mittlerweile mehren sich auch die Hinweise, dass eine nur geringe Erhöhung

\title{
Körperbeherrschung gegen Herzschmerzen
}

Den Bewegungsmangel zu beheben, an dem viele Herzpatienten leiden, ist nicht einfach. Die chinesischen Bewegungstrainings Qigong und Tai-Chi können dabei helfen.

der Pulsfrequenz bereits eine positive Auswirkung auf die Risikofaktoren für Herz-Kreislauf-Erkrankungen hat [1].

Für Herzpatienten ist Tai-Chi eine sichere Bewegungstherapie. Das gilt auch für solche, die bereits einen Bypass haben, einen Myokard-Infarkt erlitten haben oder ein Herzversagen hatten - sie können Tai-Chi-Übungen ohne grösseres Risiko absolvieren. Der direkte Nutzen für die Patienten ist eine Reduktion von koronaren Risikofaktoren wie etwa Bluthochdruck oder Dyslipidämie: Tai-Chi ist somit eine gute alternative Bewegungstherapie für Menschen, um Herz-Kreislauf-Erkrankungen zu behandeln. Daneben ist eine ganze Reihe von anderen positiven Wirkungen von Tai-Chi durch verschiedene Studien belegt. So lässt sich etwa eine Auswirkung von regelmässigen TaiChi-Übungen auf den generellen physischen und psychischen Zustand der Patienten beobachten. Tai-Chi verbessert die aerobe Kapazität und steigert die Muskelkraft, die Funktion des Endothels sowie die seelische Ausgeglichenheit.

Qigong ist eine alte chinesische Kampfsportart, die sowohl den Körper als auch den Geist trainiert und das Wohlbefinden und die Fitness steigert. Die langsamen Bewegungen, die mit Meditation und Atemrhythmus synchronisiert sind, sollen einen harmonischen Energiefluss im Kör- per auslösen. Wer Qigong über einen längeren Zeitraum ausübt, ist besser im Stressmanagement und in der Kontrolle seiner Emotionen [2]. Es gibt Hinweise, dass die Übungen die generelle Anfälligkeit für Krankheiten vermindern und im Allgemeinen dabei helfen, die Gesundheit zu erhalten. In einer Übersichtsarbeit mit 540 Patienten, die an sieben randomisiert kontrollierten Studien und einer nicht randomisiert kontrollierten Studie teilnahmen, konnten Chan et al. [2] signifikante positive Auswirkungen von Qigong bei Herzpatienten feststellen. Sie kommen zu dem Schluss, dass diese chinesische Technik vor allem für solche Patienten geeignet ist, die chronische Herzerkrankungen haben, aber nicht dazu in der Lage sind, herkömmliche westliche Fitnessprogramme zu absolvieren. Für sie bietet Qigong die Möglichkeit, belastungsarm, aber trotzdem wirkungsvoll und vor allem sicher $\mathrm{zu}$ trainieren. (rfi)

\section{Literatur}

1 Park IS, Song R, Oh KO, So HY, Kim DS Kim JI, Kim TS, Kim HL, Ahn SH: Managing cardiovascular risks with Tai Chi in people with coronary artery disease. J Adv Nurs 2010;66:282-292.

2 Chan CL, Wang CW, Ho RT, Ho AH, Ziea ET, Taam Wong VC, Ng SM: A systematic review of the effectiveness of qigong exercise in cardiac rehabilitation. Am J Chin Med 2012;40:255267.

\section{KARGER}

